\title{
NUCLOTRON BEAM DIAGNOSTICS
}

\author{
V. Andreev, V. Gorchenko, A. Govorov, A. Kirichenko, A. Kovalenko, I. Kulikov, V. Mikhailov, \\ V. Monchinsky, S. Romanov, B. Sveshnikov, A. Tsarenkov, B. Vasilishin, M. Voevodin, \\ V. Volkov\#, JINR, Dubna, Russia
}

\section{Abstract}

The superconducting synchrotron Nuclotron [1] was put into operation in March 1993 at the Laboratory of High Energies, JINR in Dubna. The Nuclotron Control System (NCS) [2] provided an efficient support for the machine operation during all runs. The dedicated NCS subsystem for beam diagnostics in the injection transfer line and the Nuclotron ring is described.

\section{INTRODUCTION}

The Nuclotron is intended to accelerate nuclei and multicharged ions up to an energy of $6 \mathrm{GeV} / \mathrm{u}$ for the charge-to-mass ratio $\mathrm{q} / \mathrm{A}=0.5$. There are 96 dipole, 64 quadrupole, 32 correcting multipole SC-magnets in the Nuclotron magnetic ring with a circumference of $251.1 \mathrm{~m}$. The maximum value of the magnetic field is about $2 \mathrm{~T}$.

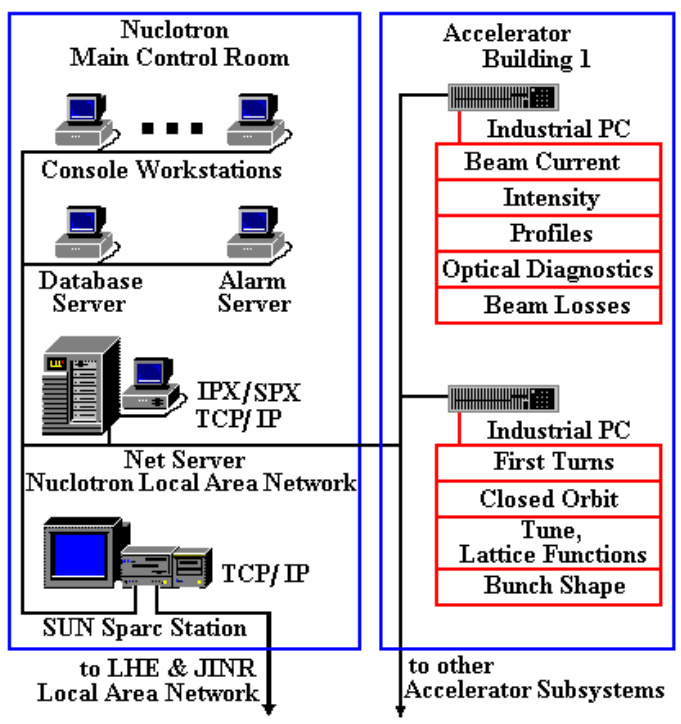

Figure 1: Fragment of the NCS structure.

The NCS consists of two physical levels: an Operator Control Level and a Front End Level. The first one supplies all appropriate man-machine tools for operators to run the accelerator. High performance workstations and server computers are used at this level.

\#Email: volkov@sunhe.jinr.ru
The workstations act as operator consoles, while the servers provide a communication process, data storage, printing utilities, a common database, alarm service, a program library, and data exchange between the Nuclotron and the users. The Front End Level comprises both industrial PCs and intelligent CAMAC cratecontrollers with embedded micro-PCs. The NCS is a distributed system. Its subsystems are geographically separated by as much as $500 \mathrm{~m}$. The common backbone of the system is an Ethernet Local Area Network.

The beam diagnostics subsystem integrated into the NCS (Fig. 1) has been operating successfully since the beginning of the Nuclotron commissioning. The subsystem is based on the industrial rack-mountable PCs from ADVANTECH equipped with $\mathrm{I} / \mathrm{O}$ and communication boards.

\section{INJECTED BEAM TRANSFER LINE}

The injector (Alvaretz type linac) accelerates ions with a charge-to-mass ratio of $0.28<\mathrm{q} / \mathrm{A}<0.5$ up to $5 \mathrm{MeV} / \mathrm{u}$ and protons up to $20 \mathrm{MeV} / \mathrm{u}$. The injected beam transfer line $30 \mathrm{~m}$ long includes 2 bending magnets, 12 quadrupole lenses, and 12 correctors. The equipment of single-turn injection comprises a superconducting septum magnet and kick electric plates. The injected beam pulse duration $(\mathrm{q} / \mathrm{A}=0.5)$ is $8 \mu \mathrm{s}$.

Several types of detectors are used for beam diagnostics: 2 Faraday cups, 1 multi-wire beam current monitor, 2 multi-wire profilometers, 2 destructive fluorescent screen monitors, and 1 screen monitor of 95\% transparency. These instruments make beam parameter measurements available over a wide range of intensities.

The Faraday cups of $10^{6}$ charge/pulse sensitivity are used for beam intensity measurements. The IN/OUT movements are remote-controlled by means of DC motor actuators.

The beam profile monitor consists of X-and Y-wire planes. Each plane assembled on a movable frame has 32 golden tungsten wires $0.1 \mathrm{~mm}$ in diameter separated by $2 \mathrm{~mm}$. The charge-to-voltage converters (CV1...CV32, see Fig. 2) with adjustable sensitivity, sample-and-hold amplifiers (S/H1...S/H32) and a multiplexer (MPX) are placed close by the detector. A timer/synchronizer (T/S), a $40 \mathrm{kHz}$ buffered ADC of a 10-bit resolution and a multiplying scaling DAC (MDAC) are arranged in the processing center at a distance of $50 \mathrm{~m}$. The external 
trigger is provided with the machine timing system, and it is the same pulse which is driving the injection elements. The sensitivity of the profilometer is about $10^{8}$ charges/pulse.

The monitor used to measure the shape and duration of the injected beam pulse has one plane with 32 golden tungsten wires $0.05 \mathrm{~mm}$ in diameter separated by $2 \mathrm{~mm}$ and connected in parallel. A fast $50 \mathrm{MHz}$ buffered ADC of a 8-bit resolution is used for beam pulse signal digitizing. The measured data together with the parameters of the septum magnet and kick electric plates (Fig. 4) are presented to the operator to tune the injection.

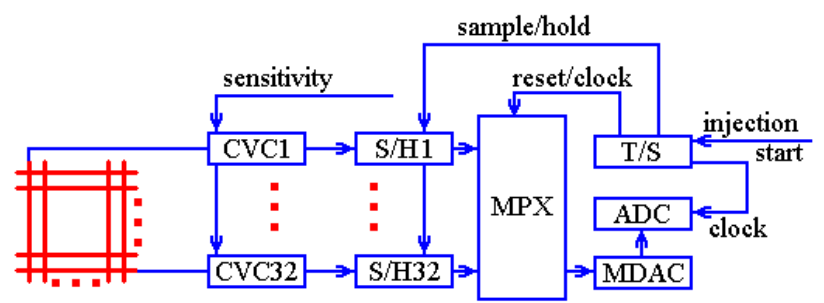

Figure 2: Profilometer block diagram.

The screen monitor of 95\% transparency (wire greed assembly) is made of horizontal and vertical tungsten wires $0.1 \mathrm{~mm}$ in diameter separated by $5 \mathrm{~mm}$ with luminophor beads in the grid junctions. The observation station is equipped with an image amplifier and a photomultiplier to register a low intensity beam current, the profile and shape of the beam pulse.

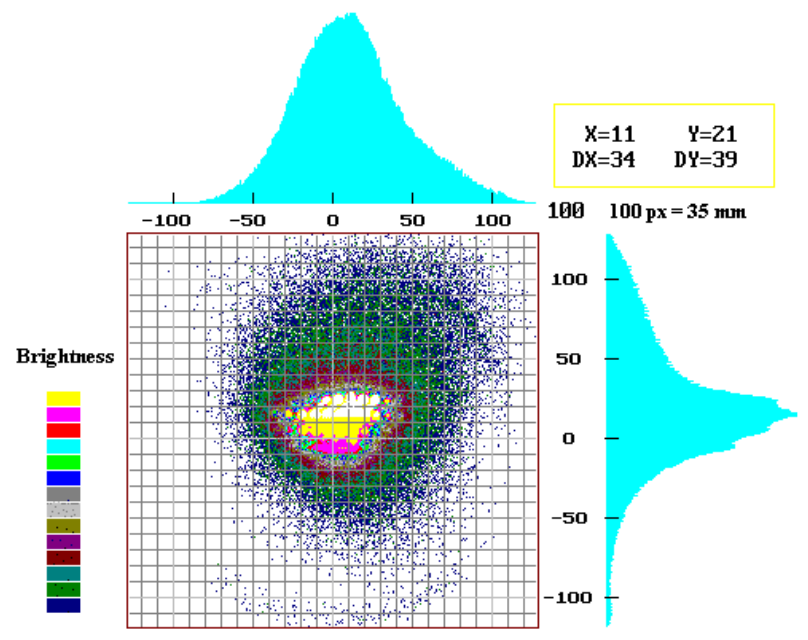

Figure 3: Beam image with X-and Y-profiles.

The image processing technique based on fluorescent screens, CCD cameras, and frame-grabbers ensures the following possibilities: screen selection and setting inside the beam, video tuning, background subtraction, pseudocolour for displays, the ability to save and restore specific images, snapshot and live mode selection (Fig. 3).

\section{NUCLOTRON RING}

\subsection{General}

The Nuclotron ring diagnostics equipment is composed of 5 multi-wire profilometers, 20 electrostatic position pick-ups, 1 electrostatic intensity pick-up, 2 beam current transformers, 4 screen monitors, and 4 Faraday cups.

The ring profilometers have the same construction and parameters as described above. Two of the five profilometers are placed at the entrance and exit of the inflector magnet. The profilometers located at the end of the transfer line, at the inflector magnet entrance, and in the accelerator ring straight section permit one to measure the injected beam emittance and to adjust more exactly injected beam matching to the ring lattice.

The localization of beam losses around the accelerator ring facilitates machine tuning. Therefore, mounting beam loss monitors and developing data acquisition electronics are planned.

\subsection{Intensity Monitors}

The intensity and shape of the bunches (Fig. 4) are measured with a special-purpose electrostatic pick-up. The corresponding amplifier has a bandwidth of $30 \mathrm{MHz}$ and a controllable gain within a range of $60 \mathrm{~dB}$. The base line restorer insures the specified output level between bunch signals. This device covers a beam intensity from $10^{9}$ to $10^{12}$ charges per pulse. The average beam intensity signal is digitized with a 12-bit ADC after analog processing.

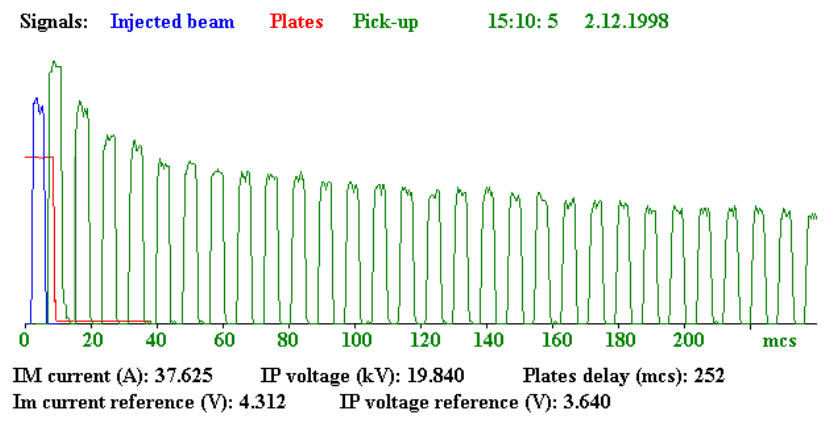

Figure 4: Example of the bunch intensity and shape measurement during first turns (RF off).

Additional opportunities for beam diagnostics are maintained by beam current transformers. To observe the longitudinal profile of the bunched beam, a fast current transformer (a frequency band of $50 \mathrm{kHz} . .10 \mathrm{MHz}$ ) is used. The transformer toroid core is made of thin (0.02 $\mathrm{mm}$ ) high permeability alloy tape with an amorphous structure. There are one sense 30-turn winding connected to the amplifier and one 1-turn test winding allowing a calibration signal to be fed in. The measured beam current ranges from 0.1 to $10 \mathrm{~mA}$. 
The DC current transformer is employed for circulating beam average current measurements. It consists of 2 cores with 2 exciting, 1 measuring, and 1 calibration windings. The exciting frequency of the modulator is $25 \mathrm{kHz}$. A filter together with a demodulator and an integrator is used for signal secondharmonic selection and its conversion into a DC voltage. The sensitivity of the device is $200 \mathrm{~V} / \mathrm{A}$, and the frequency band is $0 \ldots 60 \mathrm{kHz}$.

\subsection{Beam Position Monitors}

20 BPM electrostatic pick-ups in the form of diagonally cut $66(\mathrm{H}) \times 132(\mathrm{~W}) \times 110(\mathrm{~L}) \mathrm{mm}$ boxes are arranged in the Nuclotron ring for non-destructive measurements of the beam position along the machine. The BPM front end amplifiers are placed close by the pick-ups but outside the vacuum chamber and have a room temperature. The processing electronics are located in the centre of the ring so that the longest cable segment is smaller than 50 $\mathrm{m}$. This allows us to have a precise signal matching and negligible attenuation with relatively inexpensive cable. The main purpose of the BPM hardware and software is to provide accurate information for the correction of orbit errors. Some results obtained with the BPM system are presented in Fig. 5. The dashed line is the original closed horizontal orbit without correction at the field of injection. The solid line is drawn after correction.

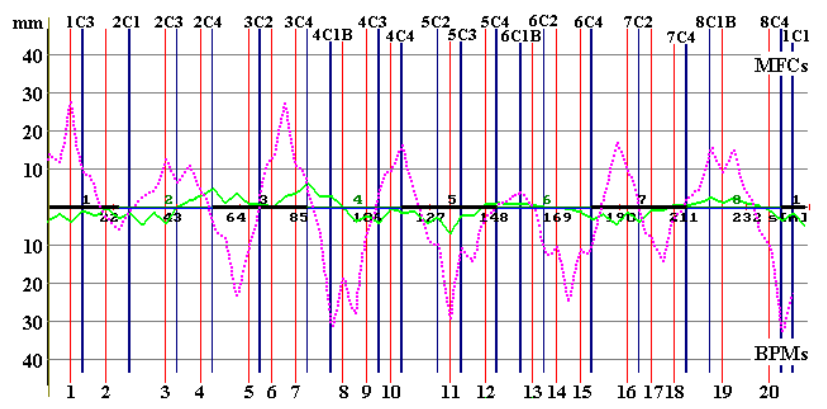

Figure 5: X-closed orbit before and after correction.

At present, we are redesigning the BPM electronics and post-processing software to extend functionality and to improve processing speed, dynamic range, and resolution. In accordance with specification, the BPM system has to function in a first turn (turns) mode and in a closed orbit mode. A substantial hardware upgrade enables one to acquire beam signals with turn-by-turn and bunch-by-bunch capabilities.

The distinguishing features of the new BPM system are outlined in Fig. 6. Each position monitor electrode has a $30 \mathrm{MHz}$ amplifier with an adjustable gain of $46 \mathrm{~dB}$ for use with high and low intensity beams. A gain control loop keeps a maximum signal level to improve the signal-to-noise ratio. For cost reasons, the amplifier has one pair ( $\mathrm{X}$ or $\mathrm{Z}$ ) of remotely selected output signals.
The beam revolution frequency $\left(\mathrm{f}_{\mathrm{rev}}\right)$ ranges from 125 $\mathrm{kHz}$ to $1.2 \mathrm{MHz}$. The accelerating frequency harmonic number is 5 . The timer/synchronizer uses a B-train with a 0.1 Gs resolution and $f_{R F}=5 f_{\text {rev }}$ as an external clock to synchronize with the main magnetic field and bunch signal. An $\mathrm{ADC} 1 \ldots \mathrm{ADC} 40$ sample rate of $50 \mathrm{MS} / \mathrm{s}$ ensures the required signal resolution over the band up to $500 \mathrm{kHz}$. A simultaneous sampling of all the BPMstations with a $64 \mathrm{~KB}$ record length permits acquiring several hundred successive closed orbits. Waveform digitizers (DSO-2125 from CyberResearch) with a 256 KB onboard memory and a time-bin duration of $4 \mathrm{~ns}$ allow more than 1000 turns to be acquired under combined parallel/sequential sampling.

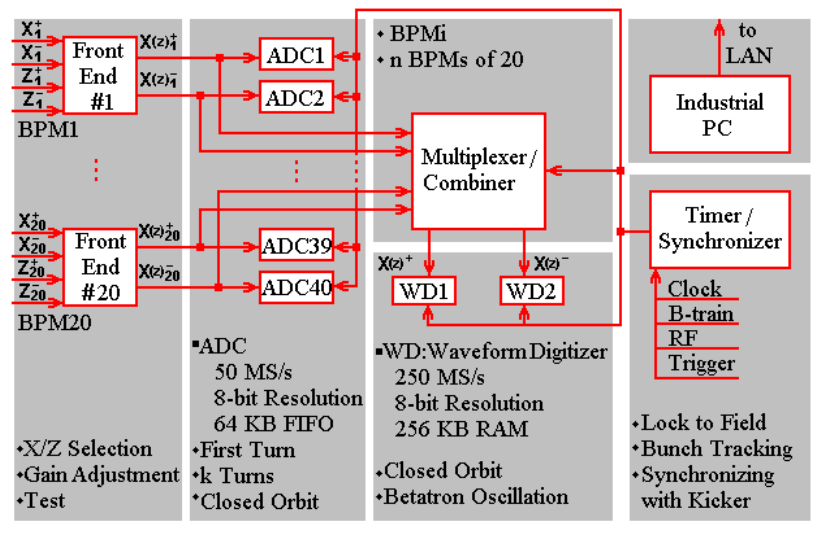

Figure 6: Structural model of BPM hardware.

The analysis of the acquired data is a very powerful tool to provide a reliable measurement of the tunes and lattice parameters of the real machine, such as beta values, or a phase advance between BPMs.

\section{CONCLUSION}

The beam diagnostics subsystem has been successfully used in all Nuclotron runs. It ensures reliable operating conditions for different operating modes of the accelerator. Several modifications are still under way in order to improve the beam diagnostics functionality. New information provided with the subsystem will be a significant aid to optimize the machine.

\section{ACKNOWLEDGEMENTS}

We would like to thank our colleagues who have contributed to the Nuclotron beam diagnostics development. The authors are grateful to L. Sveshnikova for her help in preparing this paper.

\section{REFERENCES}

[1] A. D. Kovalenko, "Status of the Nuclotron", EPAC'94, London, (1994) [2] V. Andreev et al, "Nuclotron Control System", PAC'97 Vancouver, (1997) 